\title{
EL CÁNCER DE PRÓSTATA EN LA COMUNIDAD DE MADRID EN EL AÑO 2000. II - PRESENTACIÓN Y DIAGNÓSTICO $(*)$
}

\author{
F. HERRANZ AMO ${ }^{1}$, F. ARIAS FUNEZ ${ }^{2}$, M. ARRIZABALAGA MORENO ${ }^{3}$, \\ F.J. CALAHORRA FERNÁNDEZ ${ }^{4}$, J. CARBALLIDO RODRÍGUEZ ${ }^{5}$, R. DIZ RODRÍGUEZ ${ }^{6}$, \\ J.A. HERRERO PAYO ${ }^{7}$, C. LLORENTE ABARCA ${ }^{8}$, J.C. MARTÍN MARTÍNEZ ${ }^{9}$, \\ L. MARTÍNEZ-PIÑEIRO LORENZO ${ }^{10}$, R. MÍNGUEZ MARTÍNEZ ${ }^{11}$, J. MORENO \\ SIERRA $^{12}$, A. RODRÍGUEZ ANTOLÍN ${ }^{13}$, J.C. TAMAYO RUIZ ${ }^{14}$, J. TURO ANTONA ${ }^{15}$
}

${ }^{1}$ H.G.U. Gregorio Marañón. ${ }^{2} \mathrm{H}$. Ramón y Cajal. ${ }^{3} \mathrm{H}$. de Móstoles. ${ }^{4}$ Fundación Jiménez Díaz. ${ }^{5} \mathrm{H}$. Puerta de Hierro. ${ }^{6} \mathrm{H}$. del Aire. ${ }^{7} \mathrm{H}$. Severo Ochoa. ${ }^{8} \mathrm{H}$. Fundación de Alcorcón. ${ }^{9} \mathrm{H}$. El Escorial. ${ }^{10} \mathrm{H}$. de La Paz. ${ }^{11} \mathrm{H}$. de La Princesa. ${ }^{12} \mathrm{H}$. Clínico. ${ }^{13} \mathrm{H} .12$ de Octubre. ${ }^{14} \mathrm{H}$. Príncipe de Asturias y ${ }^{15} \mathrm{H}$. Militar Gómez Ulla. Madrid.

Actas Urol Esp. 27 (5): 335-344, 2003

\section{RESUMEN}

"EL CÁNCER DE PRÓSTATA EN LA COMUNIDAD DE MADRID EN EL AÑO 2000. II - PRESENTACIÓN Y DIAGNÓSTICO(*)"

OBJETIVO: Conocer la forma de presentación, el método diagnóstico utilizado y el estadio clínico en el momento del diagnóstico de los sujetos diagnosticados de cáncer de próstata en la Comunidad de Madrid en el año 2000.

MATERIAL Y MÉTODOS: Se analizaron los datos de los 1.745 pacientes con diagnóstico histológico de cáncer de próstata remitidos por los 15 Hospitales que colaboraron en el estudio. Las variables analizadas fueron: patología asociada, motivo de consulta, tacto rectal, PSA, método de diagnóstico, score de Gleson agrupándose en grados de diferenciación, pruebas realizadas en el estudio de extensión tumoral y clasificación tumoral. Las variables cualitativas se expresaron en porcentajes del total de las mismas. Las variables cuantitativas se expresaron mediante la media, la desviación típica, los valores máximo y mínimo y los percentiles $25 \%, 50 \%$ (mediana) y $75 \%$.

RESULTADOS: El 67\% presentaron patología asociada. La mayoria (75\%) de los pacientes consultó por síndrome prostático. El 42,7\% presentaban al tacto rectal una glándula no sospechosa de tumor. La mitad de los pacientes en el momento del diagnóstico presentaron un PSA igual o menor a $11 \mathrm{ng} / \mathrm{ml}$. El 93\% de los sujetos fueron diagnosticados mediante biopsia transrectal ecodirigida. Los score más frecuentemente informados fueron el $6(31,3 \%)$ y el $7(28,7 \%)$. El $75 \%$ se consideraron clínicamente como enfermedad localizada a la próstata, el $12,5 \%$ como enfermedad localmente avanzada y el otro $12,5 \%$ como enfermedad metastásica.

CONCLUSIONES: La mayoria de los pacientes consultaron por sintomas no relacionados con el CP. La biopsia transrectal ecodirigida se confirma como la técnica de elección para el diagnóstico del CP. Al comparar con series históricas de nuestra Comunidad se observa una anticipación diagnóstica.

PALABRAS CLAVE: Cáncer de próstata. Presentación. Diagnóstico. Estadio tumoral.

\section{ABSTRACT}

"THE PROSTATE CANCER IN THE COMMUNITY OF MADRID IN 2000. II.- PRESENTATION AND DIAGNOSTICS"

OBJECTIVE: To know the presentation form, diagnostic method and clinical stage at the time of diagnosis in subjects with prostate cancer (PC) in the Autonomous Community of Madrid in 2000.

MATERIAL AND METHOD: Data from 1745 patients with histologically confirmed prostate cancer obtained from 15 Hospitals participating in the study was analysed. The variables studied were: associated disease, reason for visiting the hospital, digital rectal examination (DRE), PSA, diagnostic method, graded Gleason score, tests performed in the tumoral extension study and tumour staging. The qualitative variables are given in percentages of the overall number and the quantitative variables are expressed as the median, standard deviation, maximum and minimum values and $25 \%, 50 \%$ (median) and $75 \%$ percentiles.

RESULTS: $67 \%$ cases had an associated disease. In most (75\%) patients the reason for visiting the hospital was prostatic syndrome. DRE revealed that $42.7 \%$ has no tumour. At the time of diagnosis half the patients had PSA levels lower than or equal to $11 \mathrm{ng} / \mathrm{ml}$. Transrectal ultrasound-guided biopsy was used for diagnosis in 93\% subjects. The most commonly reported Gleason scores were 6 (31.3\%) and 7 (28.7\%). In $75 \%$ subjects the disease was considered to be clinically limited to the prostate, in $12.5 \%$ locally advanced and in $12.5 \%$ metastatic.

CONCLUSIONS: Most patients came to the hospital because of symptoms not related to PC. Transrectal ultrasound-guided biopsy is confirmed as the choice technique for PC diagnosis. When a comparison is made to historical series in our Autonomous Community a pattern of earlier diagnosis can be seen.

KEY WORDS: Prostate cancer. Presentation. Diagnositcs. Tumoral stage.

(*)Este artículo forma parte del estudio "El cáncer de próstata en la Comunidad de Madrid en el año 2000", auspiciado por la Sociedad Urológica Madrileña y financiado por AstraZéneca. 
Los pacientes con cáncer de próstata $(\mathrm{CP})$ únicamente presentan síntomas específicos de su enfermedad en las fases más avanzadas de la misma. En la era pre-PSA estos síntomas se debían a las metástasis o a la invasión local por contigüidad del cuello vesical o del trígono, produciendo síntomas obstructivos infravesicales o supravesicales. Aproximadamente el $70-80 \%$ de los pacientes desarrollaban síntomas obstructivos infravesicales ${ }^{1}$, presentando un $25 \%$ retención aguda de orina ${ }^{1,2}$. Hasta en el $20 \%$ de los pacientes el diagnóstico se realizaba por los síntomas que originaban la enfermedad metastásica: dolor óseo, pérdida de peso, anemia, linfedema, sintomas neurológicos y adenopatías ${ }^{1,2}$.

La generalización en la clínica de la determinación del PSA nos permitió clasificar a los pacientes según la probabilidad de tener un $\mathrm{CP}$ en baja, moderada y elevada. Pero no fue hasta la llegada de la ecografía transrectal de alta definición y la posibilidad de realizar biopsias transrectales ecodirigidas, cuando dispusimos de la tecnología suficientemente eficaz para demostrar las sospechas adquiridas mediante la determinación del PSA.

La combinación del tacto rectal y el PSA utilizando algoritmos para decidir que sujetos son sometidos a biopsia prostática, ha aumentado de forma espectacular el número de diagnósticos de $\mathrm{CP}$ y por lo tanto su incidencia. Pero además está modificando la forma de presentación y el estadio tumoral en el momento del diagnóstico.

En este artículo presentamos los datos sobre presentación, método diagnóstico y clasificación tumoral extraídos del estudio: "El cáncer de próstata en la Comunidad de Madrid en el año 2000".

\section{MATERIAL Y MÉTODO}

El ámbito del estudio y la metodología es la misma que la descrita en el artículo anterior: "El cáncer de próstata en la Comunidad de Madrid en el año 2000. I - Incidencia".

El objetivo de este artículo es describir la forma de presentación de los sujetos diagnosticados de CP (síntomas, niveles de PSA y tacto rectal), el método diagnóstico utilizado para su diagnóstico histológico y el estadio clínico en el momento del diagnóstico.
Según los datos sobre el estudio de extensión aportados por los Investigadores, los pacientes se clasificaron según la clasificación TNM de la UICC de $1997^{3}$. Los pacientes fueron agrupados en:

1. Enfermedad localizada, donde se incluyeron todos los pacientes clasificados como T1-2 (enfermedad glandular intracapsular), sin afectación ganglionar conocida ( $\mathrm{NO} / \mathrm{Nx})$ y sin metástasis conocidas $(\mathrm{MO} / \mathrm{Mx})$.

2. Enfermedad localmente avanzada, se incluyeron todos los pacientes clasificados como T3-4 (enfermedad glandular extracapsular), NO/Nx, $\mathrm{MO} / \mathrm{Mx}$.

3 Enfermedad metastásica, se incluyeron todos los pacientes T1-4, con afectación ganglionar (N1-3) o metástasis en otros órganos, fundamentalmente óseas (M1).

Las variables cualitativas se expresaron en porcentajes del total de las mismas. Las variables cuantitativas se expresaron mediante la media, la desviación típica, los valores máximo y mínimo y los percentiles $25 \%, 50 \%$ (mediana) y $75 \%$.

El análisis de los datos se realizó para los 1.745 pacientes de los 15 hospitales que remitieron sus datos. Se evaluaron las siguientes variables: patología asociada, motivo de consulta, tacto rectal, PSA, método de diagnóstico, score de Gleason agrupándose en grados de diferenciación: bien (score 2-4), moderadamente (score 5-7) y pobremente diferenciado (score 8-10), pruebas realizadas en el estudio de extensión tumoral y clasificación tumoral. En cada apartado se especifica el número de pacientes evaluables.

\section{RESULTADOS}

Patología asociada (1.011 pacientes evaluables - 58\%). El 67\% (673) de los casos presentaron patología asociada, siendo ésta múltiple en el $36 \%$ (239) de los pacientes. Las patologías asociadas más frecuentes fueron: digestiva 30\%, pulmonar $29 \%$, cardíaca $28 \%$, vascular $27 \%$, diabetes $17 \%$ y neurológica $9 \%$. $\mathrm{Al}$ analizar la distribución de la patología asociada según las Áreas Sanitarias, entre el $47 \%$ y el $95 \%$ de los pacientes con CP presentaban alguna otra patología asociada: el $11 \%-53 \%$ cardíaca, el 7\%-45\% pulmonar, el 21\%-40\% digestiva, el 3\%-14\% neurológica; entre el 0\% y el 51\% vascular y entre el $9 \%$ y el $27 \%$ diabetes. La relación pormenorizada por Áreas Sanitarias se detallan en la Tabla I. 
TABLA I

DISTRIBUCIÓN POR PATOLOGÍAS ASOCIADAS Y ÁREAS SANITARIAS DE LOS PACIENTES DIAGNOSTICADOS DE CÁNCER DE PRÓSTATA EN EL AÑO 2000 EN LA COMUNIDAD DE MADRID (FALTAN LAS ÁREAS 5 Y 11 QUE NO APORTARON DATOS Y EL ÁREA 10 QUE NO PARTICIPÓ EN EL ESTUDIO).

\begin{tabular}{||c|c|c|c|c|c|c|c|c|c||}
\hline & N & PE (\%) & PA (\%) & PC (\%) & PP (\%) & PD (\%) & PN (\%) & PV (\%) & DM (\%) \\
\hline A1 & 228 & $207(91 \%)$ & $112(54 \%)$ & $21(19 \%)$ & $50(45 \%)$ & $28(25 \%)$ & $14(13 \%)$ & $4(4 \%)$ & $21(19 \%)$ \\
\hline A2 & 88 & $86(98 \%)$ & $64(74 \%)$ & $15(23 \%)$ & $19(30 \%)$ & $23(36 \%)$ & $6(9 \%)$ & $28(44 \%)$ & $11(17 \%)$ \\
\hline A3 & 81 & $59(73 \%)$ & $56(95 \%)$ & $6(11 \%)$ & $13(23 \%)$ & $21(38 \%)$ & $4(7 \%)$ & $23(41 \%)$ & $12(21 \%)$ \\
\hline A4 & 304 & $227(75 \%)$ & $155(68 \%)$ & $39(25 \%)$ & $44(28 \%)$ & $54(35 \%)$ & $17(11 \%)$ & $67(43 \%)$ & $23(15 \%)$ \\
\hline A6 & 84 & $84(100 \%)$ & $53(63 \%)$ & $15(29 \%)$ & $22(42 \%)$ & $12(23 \%)$ & $3(6 \%)$ & $20(38 \%)$ & $7(14 \%)$ \\
\hline A7 & 142 & $132(93 \%)$ & $86(65 \%)$ & $38(44 \%)$ & $24(28 \%)$ & $18(21 \%)$ & $9(11 \%)$ & $9(11 \%)$ & $8(9 \%)$ \\
\hline A8 & 144 & $57(40 \%)$ & $27(47 \%)$ & $41(15 \%)$ & $2(7 \%)$ & $6(22 \%)$ & $1(4 \%)$ & 0 & $5(19 \%)$ \\
\hline A9 & 75 & $63(84 \%)$ & $53(84 \%)$ & $28(44 \%)$ & $8(13 \%)$ & $15(24 \%)$ & $2(3 \%)$ & $6(10 \%)$ & $9(14 \%)$ \\
\hline A12 & 70 & $57(81 \%)$ & $37(65 \%)$ & $10(27 \%)$ & $7(19 \%)$ & $14(38 \%)$ & $5(14 \%)$ & $19(51 \%)$ & $9(24 \%)$ \\
\hline H.M. & 46 & $39(85 \%)$ & $30(77 \%)$ & $15(50 \%)$ & $7(23 \%)$ & $12(40 \%)$ & $1(3 \%)$ & $4(13 \%)$ & $8(27 \%)$ \\
\hline
\end{tabular}

Motivo de consulta (1.116 pacientes evaluables - 64\%). Síndrome prostático 75\%, elevación del PSA $16 \%$, dolores óseos $2,3 \%$, síndrome paraneoplásico $1,7 \%$ y otros motivos de consulta $5 \%$. Al analizar la distribución por Áreas encontramos que el 55\%-100\% de los pacientes consultaron por síndrome prostático, el 8\%-36\% por elevación del PSA, el 1\%-5\% por dolor óseo, el 1\%-8,4\% por síndrome paraneoplásico y el 2\%-13\% por otros síntomas urológicos. La relación pormenorizada por Áreas Sanitarias se detallan en la Tabla II.
Tacto rectal (1.141 pacientes evaluables - 65\%). El 47,2\% de los pacientes presentaban una glándula prostática no sospechosa al tacto, el 36,9\% un nódulo localizado a la próstata, el 12,7\% un nódulo con extensión fuera de la próstata y el $3,2 \%$ una próstata pétrea con fijación de la misma a las estructuras vecinas. Las dos últimas categorías las podemos agrupar como localmente avanzada al tacto rectal (15,9\%). Al analizar la distribución por áreas, entre el 8\% (hospitales militares) y el 76\% (área 4) de los pacientes pre-

\section{TABLA II}

DISTRIBUCIÓN POR MOTIVO DE CONSULTA Y ÁREAS SANITARIAS DE LOS PACIENTES DIAGNOSTICADOS DE

CÁNCER DE PRÓSTATA EN EL AÑO 2000 EN LA COMUNIDAD DE MADRID (FALTAN LAS ÁREAS 5 Y 11 QUE NO APORTARON DATOS Y EL ÁREA 10 QUE NO PARTICIPÓ EN EL ESTUDIO).

\begin{tabular}{||c|c|c|c|c|c|c|c||}
\hline & N & PE (\%) & SP (\%) & DO (\%) & SPN (\%) & PSA (\%) & Otros (\%) \\
\hline A1 & 228 & $213(93 \%)$ & $183(86 \%)$ & $3(1 \%)$ & $4(2 \%)$ & $17(8 \%)$ & $6(3 \%)$ \\
\hline A2 & 88 & $88(100 \%)$ & $88(100 \%)$ & - & - & - & - \\
\hline A3 & 81 & $70(86 \%)$ & $56(80 \%)$ & $3(4 \%)$ & $3(4 \%)$ & $6(9 \%)$ & $2(3 \%)$ \\
\hline A4 & 304 & $223(73 \%)$ & $162(73 \%)$ & $5(2 \%)$ & $2(1 \%)$ & $36(16 \%)$ & $18(8 \%)$ \\
\hline A6 & 84 & $84(100 \%)$ & $46(55 \%)$ & $3(4 \%)$ & $2(2 \%)$ & $30(36 \%)$ & $3(4 \%)$ \\
\hline A7 & 142 & $127(89 \%)$ & $85(67 \%)$ & $5(4 \%)$ & $2(2 \%)$ & $19(15 \%)$ & $16(13 \%)$ \\
\hline A8 & 144 & $140(97 \%)$ & $90(64 \%)$ & $3(2 \%)$ & - & $38(27 \%)$ & $9(6 \%)$ \\
\hline A9 & 75 & $71(95 \%)$ & $54(76 \%)$ & $1(1,4 \%)$ & $6(8 \%)$ & $9(13 \%)$ & $1(1,4 \%)$ \\
\hline A12 & 70 & $57(82 \%)$ & $40(70 \%)$ & $1(2 \%)$ & - & $15(26 \%)$ & $1(2 \%)$ \\
\hline H.M. & 46 & $43(93 \%)$ & $31(72 \%)$ & $2(5 \%)$ & - & $9(21 \%)$ & $1(2 \%)$ \\
\hline
\end{tabular}

N: número total de casos. PE: pacientes evaluables. SP: sindrome prostático. DO: dolores óseos.

SPN: sindrome paraneoplásico. PSA: elevación del PSA. HM: hospitales militares. 
sentaban una próstata no sospechosa al tacto rectal. El 73\%-92\% fue clasificado como enfermedad localizada al tacto rectal y el $8 \%-27 \%$ como enfermedad localmente avanzada. La relación pormenorizada por Áreas Sanitarias se detallan en la Tabla III.

Antígeno específico prostático (1.567 pacientes evaluables - 90\%). El PSA medio fue de 75,9 $\pm 489,5(0,7-12,705) \mathrm{ng} / \mathrm{ml}$. El percentil del $25 \%$ fue de 7 , el del $50 \%$ de 11 y el del $75 \%$ de $24 \mathrm{ng} / \mathrm{ml}$. La media de PSA más baja se observó en los Hospitales Militares $(22 \pm 31 \mathrm{ng} / \mathrm{ml}$. con una mediana de 11) y la más elevada en el área 7 (167 $\pm 950 \mathrm{ng} / \mathrm{ml}$, con una mediana de 16). En las áreas 4,5 , y 8 más de la mitad de sus pacientes tenían menos de $10 \mathrm{ng} / \mathrm{ml}$ de PSA en el momento del diagnóstico. Los resultados pormenorizados por Áreas Sanitarias se detallan en la Tabla IV.

Método de diagnóstico (1.664 pacientes evaluables - 95\%). El 93\% de los pacientes fue diagnosticado con biopsia transrectal dirigida por ultrasonidos, el 3\% con biopsia digito-dirigida y el 4\% se diagnosticó de forma incidental a raíz de otras cirugias. La biopsia transrectal dirigida mediante ecografia fue el método de diagnóstico más frecuente, entre el 59\% en el área 6 y el 100\% en el área 11 . Se realizó biopsia "ciega" o digito-dirigida entre el 0\% y el 35\% (Área 6). El CP se diagnosticó de forma incidental debido a cirugía prostática

\section{TABLA III}

DISTRIBUCIÓN SEGÚN LOS HALLAZGOS AL TACTO RECTAL Y ÁREAS SANITARIAS DE LOS PACIENTES

DIAGNOSTICADOS DE CÁNCER DE PRÓSTATA EN EL AÑO 2000 EN LA COMUNIDAD DE MADRID (FALTAN LAS ÁREAS 5 Y 11 QUE NO APORTARON DATOS Y EL ÁREA 10 QUE NO PARTICIPÓ EN EL ESTUDIO)

\begin{tabular}{||c|c|c|c|c|c|}
\hline & N & PE (\%) & T. no palpable (\%) & T. localizado (\%) & T. localmente avanzado(\%) \\
\hline A1 & 228 & $218(96 \%)$ & $92(42 \%)$ & $95(44 \%)$ & $31(14 \%)$ \\
\hline A2 & 88 & $88(100 \%)$ & $26(30 \%)$ & $38(43 \%)$ & $24(27 \%)$ \\
\hline A3 & 81 & $77(95 \%)$ & $38(49 \%)$ & $25(32 \%)$ & $14(18 \%)$ \\
\hline A4 & 304 & $224(74 \%)$ & $170(76 \%)$ & $35(16 \%)$ & $8(10 \%)$ \\
\hline A6 & 84 & $84(100 \%)$ & $42(50 \%)$ & $34(40 \%)$ & $25(19 \%)$ \\
\hline A7 & 142 & $132(93 \%)$ & $37(28 \%)$ & $70(53 \%)$ & $27(19 \%)$ \\
\hline A8 & 144 & $141(98 \%)$ & $71(50 \%)$ & $43(30 \%)$ & $18(25 \%)$ \\
\hline A9 & 75 & $72(96 \%)$ & $21(29 \%)$ & $33(46 \%)$ & $9(14 \%)$ \\
\hline A12 & 70 & $65(93 \%)$ & $38(58 \%)$ & $18(28 \%)$ & $7(18 \%)$ \\
\hline H.M. & 46 & $40(87 \%)$ & $3(8 \%)$ & $30(75 \%)$ & \\
\hline
\end{tabular}

N: número total de casos. PE: pacientes evaluables. HM: hospitales militares.

\section{TABLA IV}

NIVELES DE PSA (en ng/ml) EN EL MOMENTO DEL DIAGNÓSTICO EN LOS PACIENTES CON CÁNCER DE PRÓSTATA EN LA COMUNIDAD DE MADRID EN EL AÑO 2000 SEGÚN LAS DISTINTAS ÁREAS SANITARIAS (NO APARECE EL ÁREA 10 POR NO PARTICIPAR EN EL ESTUDIO)

\begin{tabular}{|c|c|c|c|c|c|c|c|c|}
\hline Área & $\mathbf{N}$ & PE (\%) & $M$ & D.S. & Min-Máx. & P-25\% & P-50\% & P-75\% \\
\hline A1 & 228 & 218 (96\%) & 64 & 170 & $2-1054$ & 7 & 12 & 29 \\
\hline A2 & 88 & 88 (100\%) & 94 & 263 & 5-1510 & 7 & 15 & 42 \\
\hline A3 & 81 & 76 (94\%) & 139 & 601 & $3-4434$ & 8 & 15 & 30 \\
\hline A4 & 304 & 227 (75\%) & 25 & 67 & $1-593$ & 6 & 8 & 12 \\
\hline A5 & 328 & 277 (84\%) & 57 & 334 & $2-4190$ & 6 & 9 & 18 \\
\hline A6 & 84 & 84 (100\%) & 43 & 106 & 4-745 & 8 & 13 & 24 \\
\hline A7 & 142 & $131(92 \%)$ & 167 & 950 & 4-9926 & 10 & 16 & 36 \\
\hline A8 & 144 & $140(97 \%)$ & 125 & 1075 & $1-12705$ & 6 & 8 & 17 \\
\hline A9 & 75 & 74 (99\%) & 59 & 155 & $1-882$ & 7 & 10 & 25 \\
\hline A11 & 155 & 147 (95\%) & 71 & 220 & $2-2100$ & 9 & 14 & 48 \\
\hline A12 & 70 & 65 (93\%) & 89 & 457 & $2-3680$ & 7 & 10 & 28 \\
\hline H.M. & 46 & 40 (87\%) & 22 & 31 & 4-166 & 8 & 11 & 17 \\
\hline
\end{tabular}

$\mathrm{N}$ : número total de casos. PE: pacientes evaluables, porcentaje entre paréntesis. M: media de PSA. D.S.: desviación estándar. Min-máx.: valores minimo y máximo. P: percentiles al 25\%, 50\% (mediana) y 75\%. HM: hospitales militares. 
entre el $1 \%$ en el área 2 y el $12 \%$ en los hospitales militares. La relación pormenorizada por Áreas Sanitarias se detallan en la Tabla V.

Score de Gleason (1.719 pacientes evaluables $98,5 \%$ ). Score $21,3 \%$, score $31,3 \%$, score $45,5 \%$, score $59,9 \%$, score $631,3 \%$, score $728,7 \%$, score $811,2 \%$, score $97,2 \%$, score $101,2 \%$ y no valorables $2,4 \%$. La distribución por Áreas Sanitarias del score de Gleason se detalla en la Tabla VI.
Grados de diferenciación (1.692 pacientes evaluables - 97\%). Bien diferenciado (score 2-4) 9\%, moderadamente diferenciado (score 5-7) $71 \%$ y pobremente diferenciado (score 8-10) 20\%. Las áreas $1,2,4,5$ y 11 tienen un porcentaje de casos de bien diferenciado muy pequeño (entre $0 \%$ y $5 \%$ ) en comparación con las áreas $3,6,7,8,9,12$ y Hospitales Militares (entre 18\% y 24\%). La distribución por Áreas se detalla en la Tabla VII.

\section{TABLA V}

DISTRIBUCIÓN SEGÚN EL MÉTODO UTILIZADO PARA EL DIAGNÓSTICO Y ÁREAS SANITARIAS DE LOS PACIENTES DIAGNOSTICADOS DE CÁNCER DE PRÓSTATA EN EL AÑO 2000 EN LA COMUNIDAD DE MADRID (NO APARECE EL ÁREA 10 POR NO PARTICIPAR EN EL ESTUDIO)

\begin{tabular}{||l|c|c|c|c|c|}
\hline & $\mathbf{N}$ & PE (\%) & BED (\%) & BC (\%) & CP (\%) \\
\hline A1 & 228 & $222(97 \%)$ & $214(96 \%)$ & - & $8(4 \%)$ \\
\hline A2 & 88 & $87(99 \%)$ & $86(99 \%)$ & - & $1(1 \%)$ \\
\hline A3 & 81 & $68(84 \%)$ & $48(71 \%)$ & $14(21 \%)$ & $6(9 \%)$ \\
\hline A4 & 304 & $303(99,7 \%)$ & $286(94 \%)$ & $1(0,3 \%)$ & $8(5 \%)$ \\
\hline A5 & 328 & $328(100 \%)$ & $320(98 \%)$ & - & $5(6 \%)$ \\
\hline A6 & 84 & $82(98 \%)$ & $48(59 \%)$ & $29(35 \%)$ & $5(5 \%)$ \\
\hline A7 & 142 & $101(71 \%)$ & $93(92 \%)$ & $6(3 \%)$ & $4(5 \%)$ \\
\hline A8 & 144 & $144(100 \%)$ & $131(91 \%)$ & - & - \\
\hline A9 & 75 & $70(93 \%)$ & $66(94 \%)$ & - & $1(1,5 \%)$ \\
\hline A11 & 155 & $155(100 \%)$ & $155(100 \%)$ & $1,5 \%)$ & $5(12 \%)$ \\
\hline A12 & 70 & $61(87 \%)$ & $59(97 \%)$ & - & \\
\hline H.M. & 46 & $43(93 \%)$ & $38(88 \%)$ & & $5)$ \\
\hline
\end{tabular}

N: número de casos. PE: pacientes evaluables. BED: biopsia ecodirigida. BC: biopsia "ciega". CP: cirugia prostática. HM: hospitales militares.

\section{TABLA VI}

DISTRIBUCIÓN DEL SCORE DE GLEASON ENCONTRADO EN LA BIOPSIA SEGÚN LAS DISTINTAS ÁREAS SANITARIAS DE LOS PACIENTES DIAGNOSTICADOS DE CÁNCER DE PRÓSTATA EN EL AÑO 2000 EN LA COMUNIDAD DE MADRID, EL PORCENTAJE SE EXPRESA ENTRE PARÉNTESIS (NO APARECE EL ÁREA 10 POR NO PARTICIPAR EN EL ESTUDIO)

\begin{tabular}{|c|c|c|c|c|c|c|c|c|c|c|c|c|}
\hline & $\mathbf{N}$ & PE & NV & S-2 & S-3 & S-4 & S-5 & S-6 & S-7 & S-8 & S-9 & S-10 \\
\hline A1 & 228 & $\begin{array}{c}219 \\
(96 \%)\end{array}$ & $\begin{array}{c}3 \\
(1,4 \%)\end{array}$ & - & $\begin{array}{c}1 \\
(0,5 \%)\end{array}$ & $\begin{array}{c}4 \\
(1,8 \%)\end{array}$ & $\begin{array}{c}3 \\
(1,4 \%)\end{array}$ & $\begin{array}{c}74 \\
(33,8 \%) \\
\end{array}$ & $\begin{array}{c}67 \\
(30,6 \%) \\
\end{array}$ & $\begin{array}{c}43 \\
(19,6 \%) \\
\end{array}$ & $\begin{array}{c}23 \\
(10,5 \%) \\
\end{array}$ & $\begin{array}{c}1 \\
(0,5 \%)\end{array}$ \\
\hline A2 & 88 & $\begin{array}{c}88 \\
(100 \%)\end{array}$ & - & - & - & $\begin{array}{c}1 \\
(1,1 \%)\end{array}$ & $\begin{array}{c}5 \\
(5,7 \%)\end{array}$ & $\begin{array}{c}36 \\
(40,9 \%)\end{array}$ & $\begin{array}{c}26 \\
(29,5 \%)\end{array}$ & $\begin{array}{c}12 \\
(13,6 \%)\end{array}$ & $\begin{array}{c}7 \\
(8 \%)\end{array}$ & $\begin{array}{c}1 \\
(1,1 \%)\end{array}$ \\
\hline A3 & 81 & $\begin{array}{c}77 \\
(95 \%)\end{array}$ & $\begin{array}{c}3 \\
(3,9 \%)\end{array}$ & $\begin{array}{c}4 \\
(5,2 \%)\end{array}$ & $\begin{array}{c}6 \\
(7,8 \%)\end{array}$ & $\begin{array}{c}5 \\
(6,5 \%)\end{array}$ & $\begin{array}{c}19 \\
(24,7 \%)\end{array}$ & $\begin{array}{c}10 \\
(13 \%)\end{array}$ & $\begin{array}{c}18 \\
(23,4 \%)\end{array}$ & $\begin{array}{c}8 \\
(10,4 \%)\end{array}$ & $\begin{array}{c}3 \\
(3,9 \%)\end{array}$ & $\begin{array}{c}1 \\
(1,3 \%)\end{array}$ \\
\hline A4 & 304 & $\begin{array}{c}304 \\
(100 \%)\end{array}$ & $\begin{array}{c}1 \\
(0,3 \%)\end{array}$ & - & - & $\begin{array}{c}2 \\
(0,7 \%) \\
\end{array}$ & $\begin{array}{c}14 \\
(4,6 \%)\end{array}$ & $\begin{array}{c}76 \\
(25 \%) \\
\end{array}$ & $\begin{array}{c}157 \\
(51,6 \%) \\
\end{array}$ & $\begin{array}{c}24 \\
(7,9 \%) \\
\end{array}$ & $\begin{array}{c}30 \\
(9,9 \%) \\
\end{array}$ & - \\
\hline A5 & 328 & $\begin{array}{c}328 \\
(100 \%)\end{array}$ & $\begin{array}{c}5 \\
(1,5 \%)\end{array}$ & $\begin{array}{c}3 \\
(0,9 \%)\end{array}$ & $\begin{array}{c}1 \\
(0,3 \%)\end{array}$ & $\begin{array}{c}12 \\
(3,7 \%)\end{array}$ & $\begin{array}{c}16 \\
(4,9 \%)\end{array}$ & $\begin{array}{c}157 \\
(47,9 \%)\end{array}$ & $\begin{array}{c}74 \\
(22,6 \%) \\
\end{array}$ & $\begin{array}{c}26 \\
(7,9 \%) \\
\end{array}$ & $\begin{array}{c}24 \\
(7,3 \%) \\
\end{array}$ & $\begin{array}{c}10 \\
(3 \%)\end{array}$ \\
\hline A6 & 84 & $\begin{array}{c}84 \\
(100 \%) \\
\end{array}$ & $\begin{array}{c}2 \\
(2,4 \%)\end{array}$ & $\begin{array}{c}1 \\
(1,2 \%)\end{array}$ & $\begin{array}{c}3 \\
(3,6 \%) \\
\end{array}$ & $\begin{array}{c}10 \\
(11,9 \%)\end{array}$ & $\begin{array}{c}18 \\
(21,4 \%)\end{array}$ & $\begin{array}{c}20 \\
(23,8 \%) \\
\end{array}$ & $\begin{array}{c}17 \\
(20,2 \%) \\
\end{array}$ & $\begin{array}{c}7 \\
(8,3 \%) \\
\end{array}$ & $\begin{array}{c}3 \\
(3,6 \%) \\
\end{array}$ & $\begin{array}{c}3 \\
(3,6 \%)\end{array}$ \\
\hline A7 & 142 & $\begin{array}{c}142 \\
(100 \%)\end{array}$ & $\begin{array}{c}2 \\
(1,4 \%)\end{array}$ & $\begin{array}{c}6 \\
(4,2 \%)\end{array}$ & $\begin{array}{c}4 \\
(2,8 \%)\end{array}$ & $\begin{array}{c}18 \\
(12,7 \%)\end{array}$ & $\begin{array}{c}35 \\
(24,6 \%)\end{array}$ & $\begin{array}{c}24 \\
(16,9 \%)\end{array}$ & $\begin{array}{c}40 \\
(28,2 \%)\end{array}$ & $\begin{array}{c}6 \\
(4,2 \%)\end{array}$ & $\begin{array}{c}7 \\
(4,9 \%)\end{array}$ & - \\
\hline A8 & 144 & $\begin{array}{c}141 \\
(98 \%)\end{array}$ & $\begin{array}{c}6 \\
(4,3 \%)\end{array}$ & $\begin{array}{c}1 \\
(0,7 \%)\end{array}$ & $\begin{array}{c}2 \\
(1,4 \%)\end{array}$ & $\begin{array}{c}17 \\
(12,1 \%)\end{array}$ & $\begin{array}{c}29 \\
(20,6 \%)\end{array}$ & $\begin{array}{c}22 \\
(15,6 \%)\end{array}$ & $\begin{array}{c}30 \\
(21,3 \%)\end{array}$ & $\begin{array}{c}22 \\
(15,6 \%)\end{array}$ & $\begin{array}{c}10 \\
(7,1 \%)\end{array}$ & $\begin{array}{c}2 \\
(1,4 \%)\end{array}$ \\
\hline A9 & 75 & $\begin{array}{c}74 \\
(99 \%)\end{array}$ & $\begin{array}{c}13 \\
(18 \%) \\
\end{array}$ & $\begin{array}{c}1 \\
(1,4 \%) \\
\end{array}$ & $\begin{array}{c}1 \\
(1,4 \%) \\
\end{array}$ & $\begin{array}{c}12 \\
(16,2 \%)\end{array}$ & $\begin{array}{c}9 \\
(12,2 \%)\end{array}$ & $\begin{array}{c}12 \\
(16,2 \%) \\
\end{array}$ & $\begin{array}{c}14 \\
(19,0 \%) \\
\end{array}$ & $\begin{array}{c}10 \\
(13,5 \%) \\
\end{array}$ & $\begin{array}{c}1 \\
(1,4 \%) \\
\end{array}$ & $\begin{array}{c}1 \\
(1,4 \%)\end{array}$ \\
\hline A11 & 155 & $\begin{array}{c}146 \\
(94 \%)\end{array}$ & - & - & - & - & $\begin{array}{c}5 \\
(3,4 \%) \\
\end{array}$ & $\begin{array}{c}67 \\
(45,9 \%) \\
\end{array}$ & $\begin{array}{c}36 \\
(24,7 \%) \\
\end{array}$ & $\begin{array}{c}26 \\
(17,8 \%) \\
\end{array}$ & $\begin{array}{c}12 \\
(8,2 \%) \\
\end{array}$ & - \\
\hline A12 & 70 & $\begin{array}{c}70 \\
(100 \%)\end{array}$ & $\begin{array}{c}6 \\
(8,6 \%)\end{array}$ & $\begin{array}{c}5 \\
(7,1 \%)\end{array}$ & $\begin{array}{c}5 \\
(7,1 \%)\end{array}$ & $\begin{array}{c}4 \\
(5,7 \%)\end{array}$ & $\begin{array}{c}8 \\
(11,4 \%)\end{array}$ & $\begin{array}{c}28 \\
(40 \%)\end{array}$ & $\begin{array}{c}12 \\
(17,1 \%)\end{array}$ & $\begin{array}{c}2 \\
(2,9 \%)\end{array}$ & - & - \\
\hline HM & 46 & $\begin{array}{c}46 \\
(100 \%)\end{array}$ & - & $\begin{array}{c}1 \\
(2,2 \%)\end{array}$ & - & $\begin{array}{c}9 \\
(19,6 \%)\end{array}$ & $\begin{array}{c}9 \\
(19,6 \%)\end{array}$ & $\begin{array}{c}13 \\
(28,3 \%)\end{array}$ & $\begin{array}{c}3 \\
(6,5 \%)\end{array}$ & $\begin{array}{c}6 \\
(13 \%)\end{array}$ & $\begin{array}{c}3 \\
(6,5 \%)\end{array}$ & $\begin{array}{c}2 \\
(4,3 \%)\end{array}$ \\
\hline
\end{tabular}

N: número de casos. PE: pacientes evaluables. NV: score no valorable. S: score. HM: hospitales militares. 
TABLA VII

DISTRIBUCIÓN DEL GRADO DE DIFERENCIACIÓN ENCONTRADO EN LAS BIOPSIAS SEGÚN LAS ÁREAS SANITARIAS DE LOS PACIENTES DIAGNOSTICADOS DE CÁNCER DE PRÓSTATA EN EL AÑO 2000 EN LA COMUNIDAD DE MADRID (NO APARECE EL ÁREA 10 POR NO PARTICIPAR EN EL ESTUDIO)

\begin{tabular}{||l|c|c|c|c|c||}
\hline & N & PE (\%) & G. Bajo (\%) & G. Moderado (\%) & G. Elevado (\%) \\
\hline A1 & 228 & $217(95 \%)$ & $6(3 \%)$ & $146(67 \%)$ & $65(30 \%)$ \\
\hline A2 & 88 & $88(100 \%)$ & $1(1 \%)$ & $68(77 \%)$ & $19(22 \%)$ \\
\hline A3 & 81 & $74(91 \%)$ & $15(20 \%)$ & $47(64 \%)$ & $12(16 \%)$ \\
\hline A4 & 304 & $303(99,7 \%)$ & $2(1 \%)$ & $247(81 \%)$ & $64(18 \%)$ \\
\hline A5 & 328 & $323(98 \%)$ & $16(5 \%)$ & $247(76 \%)$ & $12(15 \%)$ \\
\hline A6 & 84 & $82(98 \%)$ & $15(18 \%)$ & $55(67 \%)$ & $13(9 \%)$ \\
\hline A7 & 142 & $140(99 \%)$ & $28(20 \%)$ & $99(71 \%)$ & $32(23 \%)$ \\
\hline A8 & 144 & $136(94 \%)$ & $24(18 \%)$ & $80(59 \%)$ & $15(21 \%)$ \\
\hline A9 & 75 & $71(95 \%)$ & $18(25 \%)$ & $38(54 \%)$ & $38(26 \%)$ \\
\hline A11 & 155 & $146(94 \%)$ & - & $108(74 \%)$ & $2(3 \%)$ \\
\hline A12 & 70 & $66(94 \%)$ & $16(24 \%)$ & $48(73 \%)$ & $11(24 \%)$ \\
\hline H.M. & 46 & $46(100 \%)$ & $10(22 \%)$ & $25(54 \%)$ & \\
\hline
\end{tabular}

N: número de casos. PE: pacientes evaluables. Grado bajo. Score 2-4. Grado moderado. Score 5-7. Grado elevado 8-10. H.M: hospitales militares.

Estudio de extensión tumoral (1.127 pacientes evaluables - 65\%). Al 92\% se les realizó como mínimo una de las siguientes pruebas diagnósticas: radiografía de tórax al 34\%, urografía intravenosa al $5 \%$, ecografía abdominal al $27 \%$, tomografía computarizada (TC) abdominal al 68\%, gammagrafía ósea (GO) al $71 \%$, resonancia magnética al $2 \%$, biopsia de vesículas seminales al 0,3\%, linfadenectomía al 0,8\%, de las cuales el $89 \%$ fue mediante laparotomía, TC y GO a 677 (39\%) pacientes y TC o GO a 871 (50\%) pacientes. La relación pormenorizada por áreas y pruebas diagnósticas se detalla en la Tabla VIII.

\section{TABLA VIII}

PRUEBAS DIAGNÓSTICAS REALIZADAS EN EL ESTUDIO DE EXTENSIÓN SEGÚN LAS DISTINTAS ÁREAS SANITARIAS A LOS PACIENTES CON CÁNCER DE PRÓSTATA DIAGNOSTICADOS EN LA COMUNIDAD DE MADRID EN EL AÑO 2000 (FALTAN LAS ÁREAS 5 Y 11 QUE NO APORTARON DATOS Y EL ÁREA 10 QUE NO PARTICIPÓ EN EL ESTUDIO)

\begin{tabular}{|c|c|c|c|c|c|c|c|c|c|c|c|}
\hline & $\mathbf{N}$ & PE & RTX & UIV & ECO & TC & GO & RM & BVS & LFD & TC+GO \\
\hline A1 & 228 & $\begin{array}{c}216 \\
(95 \%)\end{array}$ & - & - & $\begin{array}{c}1 \\
(0,5 \%)\end{array}$ & $\begin{array}{c}177 \\
(82 \%)\end{array}$ & $\begin{array}{c}189 \\
(88 \%)\end{array}$ & - & - & - & $\begin{array}{c}172 \\
(80 \%)\end{array}$ \\
\hline A2 & 88 & $\begin{array}{c}88 \\
(100 \%)\end{array}$ & $\begin{array}{c}88 \\
(100 \%)\end{array}$ & - & - & $\begin{array}{c}88 \\
(100 \%)\end{array}$ & $\begin{array}{c}88 \\
(100 \%)\end{array}$ & - & - & - & $\begin{array}{c}88 \\
(100 \%)\end{array}$ \\
\hline A3 & 81 & $\begin{array}{c}77 \\
(95 \%)\end{array}$ & $\begin{array}{c}59 \\
(77 \%)\end{array}$ & $\begin{array}{c}1 \\
(1 \%)\end{array}$ & $\begin{array}{c}46 \\
(60 \%)\end{array}$ & $\begin{array}{c}33 \\
(43 \%) \\
\end{array}$ & $\begin{array}{c}59 \\
(77 \%)\end{array}$ & - & - & $\begin{array}{c}1 \\
(1 \%)\end{array}$ & $\begin{array}{c}31 \\
(40 \%)\end{array}$ \\
\hline A4 & 304 & $\begin{array}{c}227 \\
(75 \%)\end{array}$ & $\begin{array}{c}17 \\
(7 \%)\end{array}$ & $\begin{array}{c}6 \\
(3 \%)\end{array}$ & $\begin{array}{c}42 \\
(19 \%)\end{array}$ & $\begin{array}{c}174 \\
(77 \%)\end{array}$ & $\begin{array}{c}194 \\
(85 \%)\end{array}$ & $\begin{array}{c}1 \\
(0,4 \%)\end{array}$ & - & - & $\begin{array}{c}169 \\
(74 \%)\end{array}$ \\
\hline A6 & 84 & $\begin{array}{c}84 \\
(100 \%)\end{array}$ & $\begin{array}{c}56 \\
(67 \%)\end{array}$ & $\begin{array}{c}26 \\
(31 \%)\end{array}$ & $\begin{array}{c}33 \\
(39 \%)\end{array}$ & $\begin{array}{c}78 \\
(93 \%)\end{array}$ & $\begin{array}{c}78 \\
(93 \%)\end{array}$ & $\begin{array}{c}3 \\
(4 \%)\end{array}$ & - & - & $\begin{array}{c}77 \\
(92 \%)\end{array}$ \\
\hline A7 & 142 & $\begin{array}{c}131 \\
(92 \%)\end{array}$ & $\begin{array}{c}60 \\
(46 \%)\end{array}$ & $\begin{array}{c}12 \\
(9 \%)\end{array}$ & $\begin{array}{c}79 \\
(60 \%)\end{array}$ & $\begin{array}{c}43 \\
(33 \%)\end{array}$ & $\begin{array}{c}48 \\
(37 \%)\end{array}$ & $\begin{array}{c}13 \\
(10 \%)\end{array}$ & - & $\begin{array}{c}2 \\
(2 \%)\end{array}$ & $\begin{array}{c}27 \\
(21 \%)\end{array}$ \\
\hline A8 & 144 & $\begin{array}{c}138 \\
(96 \%)\end{array}$ & $\begin{array}{c}54 \\
(39 \%)\end{array}$ & $\begin{array}{c}1 \\
(1 \%)\end{array}$ & $\begin{array}{c}55 \\
(40 \%)\end{array}$ & $\begin{array}{c}48 \\
(35 \%)\end{array}$ & $\begin{array}{c}46 \\
(33 \%)\end{array}$ & - & - & - & $\begin{array}{c}28 \\
(20 \%)\end{array}$ \\
\hline A9 & 75 & $\begin{array}{c}71 \\
(95 \%)\end{array}$ & $\begin{array}{c}11 \\
(15 \%)\end{array}$ & $\begin{array}{c}4 \\
(6 \%)\end{array}$ & $\begin{array}{c}11 \\
(15 \%)\end{array}$ & $\begin{array}{c}49 \\
(69 \%)\end{array}$ & $\begin{array}{c}48 \\
(68 \%)\end{array}$ & $\begin{array}{c}1 \\
(1 \%)\end{array}$ & $\begin{array}{c}3 \\
(4 \%)\end{array}$ & $\begin{array}{c}1 \\
(1 \%)\end{array}$ & $\begin{array}{c}45 \\
(63 \%)\end{array}$ \\
\hline A12 & 70 & $\begin{array}{c}55 \\
(79 \%)\end{array}$ & $\begin{array}{c}1 \\
(1 \%)\end{array}$ & - & $\begin{array}{c}1 \\
(2 \%)\end{array}$ & $\begin{array}{c}39 \\
(71 \%)\end{array}$ & $\begin{array}{c}19 \\
(35 \%)\end{array}$ & - & - & - & $\begin{array}{c}16 \\
(29 \%)\end{array}$ \\
\hline H.M. & 46 & $\begin{array}{c}39 \\
(85 \%)\end{array}$ & $\begin{array}{c}30 \\
(77 \%)\end{array}$ & $\begin{array}{c}1 \\
(3 \%)\end{array}$ & $\begin{array}{c}37 \\
(95 \%)\end{array}$ & $\begin{array}{c}36 \\
(92 \%)\end{array}$ & $\begin{array}{c}35 \\
(90 \%)\end{array}$ & - & - & $\begin{array}{c}5 \\
(9 \%)\end{array}$ & $\begin{array}{c}34 \\
(87 \%)\end{array}$ \\
\hline
\end{tabular}

$\mathrm{N}$ : número de casos. PE: pacientes evaluables. RTx: radiografía de tórax. UIV: urografía IV. ECO: ecografỉa abdominal.

TC: tomografía computarizada. GO: gammagrafía ósea. RM: resonancia magnética. LFD: linfadenectomía.

TC+GO: pacientes a los que se les realizó TC y GO. H.M.: hospitales militares. 
Estadificación tumoral (1.139 pacientes evaluables - 65\%). Enfermedad localizada (T1-2, NO/Nx, MO/Mx) - 857 (75\%) casos. Enfermedad localmente avanzada (T3-4, NO/Nx, MO/Mx) $141(12,5 \%)$ casos. Enfermedad metastásica (T1$4, \mathrm{~N}+\mathrm{o} \mathrm{M}+)$ - $141(12,5 \%)$. La relación pormenorizada por áreas se detalla en la Tabla IX.

Relación entre el estadio tumoral y la edad (1.137 pacientes evaluables - 65\%). Enfermedad localizada 68,9 \pm 7,7 (40-94), mediana de 69 años. Enfermedad localmente avanzada 70,6 \pm 7,5 (53-86), mediana de 71 años. Enfermedad metastásica 71,2 \pm 7,8 (49-91), mediana de 71 años. Sólo las áreas 1, 3, 4, 6, 8 y 9 mantienen la relación observada en el conjunto de la $\mathrm{CM}$ en la que los casos con enfermedad localizada eran los más jóvenes (Tabla X).

\section{DISCUSIÓN}

El 67\% de los pacientes evaluados presentaban patología asociada de distinta severidad y un $36 \%$ de los mismos patología múltiple. Este elevado porcentaje de patología asociada unido a que la edad media de diagnóstico fue de 70 años, es muy probable que influyera en la sistemática del estudio de extensión tumoral y en la indicación terapéutica.

En nuestro estudio la mayoría de los pacientes consultaron por síndrome prostático $(75 \%)$ y sólo el 5\% por síntomas directamente relacionados con el CP. El 16\% presentaba únicamente elevación del PSA, existiendo importantes diferencias entre las distintas Áreas Sanitarias. En la serie de Goodman y cols. ${ }^{4}$ de pacientes diagnosticados en la época pre-PSA, el $83,8 \%$ se diagnosticaron por síntomas miccionales y el 7,3\% por dolores óseos, no se diagnosticó ningún caso en varones aparentemente sanos. Debido a que la aparición de los sintomas de HBP coincide en el tiempo con el desarrollo del $\mathrm{CP}$ es lógico que la mayoría de los pacientes con $\mathrm{CP}$ consulten por sintomas obstructivos o irritativos infravesicales. Debido a la inclusión de la determinación de PSA en los exámenes analíticos rutinarios de empresas y al aumento progresivo del "screening oportunístico" o búsqueda de casos, tanto en las consultas generalistas como en las urológicas, estamos observando el aumento de pacientes con $\mathrm{CP}$ asintomáticos diagnosticados sólo por una elevación del PSA.

El tacto rectal es una prueba diagnóstica operador dependiente, influida por la subjetividad y la experiencia del médico, y por lo tanto con una importante variabilidad interobservador. Se considera que el tacto rectal es un buen indicador del volumen tumoral en la glándula prostática, pacientes con tacto rectal no sospechoso tienen más probabilidades de tener enfermedad localizada (intra-

\section{TABLA IX}

ESTADIO TUMORAL DE LOS PACIENTES DIAGNOSTICADOS DE CÁNCER DE PRÓSTATA EN LA COMUNIDAD DE MADRID EN EL AÑO 2000, SEGÚN LAS DISTINTAS ÁREAS SANITARIAS (FALTAN LAS ÁREAS 5 Y 11 QUE NO APORTARON DATOS Y EL ÁREA 10 QUE NO PARTICIPÓ EN EL ESTUDIO).

\begin{tabular}{||l|c|c|c|c|c||}
\hline & N & PE (\%) & $\begin{array}{c}\text { E. localizada (\%) } \\
\text { avanzada (\%) }\end{array}$ & E. localmente & E. metastásica (\%) \\
\hline A1 & 228 & $217(95 \%)$ & $168(77,4 \%)$ & $20(9,2 \%)$ & $29(13,4 \%)$ \\
\hline A2 & 88 & $88(100 \%)$ & $49(55,7 \%)$ & $28(31,8 \%)$ & $11(12,5 \%)$ \\
\hline A3 & 81 & $77(95 \%)$ & $61(79,2 \%)$ & $6(7,8 \%)$ & $10(13 \%)$ \\
\hline A4 & 304 & $227(75 \%)$ & $206(90,7 \%)$ & $6(2,6 \%)$ & $15(6,6 \%)$ \\
\hline A6 & 84 & $84(100 \%)$ & $67(79,8 \%)$ & $3(3,6 \%)$ & $14(16,7 \%)$ \\
\hline A7 & 142 & $132(93 \%)$ & $90(68,2 \%)$ & $25(18,9 \%)$ & $17(12,9 \%)$ \\
\hline A8 & 144 & $137(95 \%)$ & $80(58,4 \%)$ & $36(26,3 \%)$ & $21(15,3 \%)$ \\
\hline A9 & 75 & $73(97 \%)$ & $53(72,6 \%)$ & $7(9,6 \%)$ & $13(17,8 \%)$ \\
\hline A12 & 70 & $65(93 \%)$ & $55(84,6 \%)$ & $6(9,2 \%)$ & $4(6,2 \%)$ \\
\hline H.M. & 46 & $39(85 \%)$ & $28(71,8 \%)$ & $4(10,3 \%)$ & $7(17,9 \%)$ \\
\hline
\end{tabular}

$\mathrm{N}$ : número de casos. PE: pacientes evaluables. T1-2, NO/Nx, MO/Mx: pacientes clasificados como enfermedad localizada.

T3-4, NO/Nx, MO/Mx: pacientes clasificados como enfermedad localmente avanzada.

T1-4, N+ o M+: pacientes clasificados como enfermedad metastásica. H.M.: hospitales militares. 
TABLA X

RELACIÓN ENTRE LA EDAD Y EL ESTADIO TUMORAL POR ÁREAS SANITARIAS EN LOS PACIENTES DE CÁNCER DE PRÓSTATA DIAGNOSTICADOS EN LA COMUNIDAD DE MADRID EN EL AÑO 2.000 (FALTAN LAS ÁREAS 5 Y 11 QUE NO APORTARON DATOS Y EL ÁREA 10 QUE NO PARTICIPÓ EN EL ESTUDIO)

\begin{tabular}{|c|c|c|c|c|c|c|}
\hline Área & Estadio & PE & Media & DS & Mediana & Min-Máx. \\
\hline A1 & $\begin{array}{l}\text { E. localizada } \\
\text { E. L. avanzada } \\
\text { E. metastásica }\end{array}$ & $\begin{array}{l}168 \\
20 \\
29\end{array}$ & $\begin{array}{c}69 \\
72,5 \\
71\end{array}$ & $\begin{array}{c}7 \\
6 \\
6,1\end{array}$ & $\begin{array}{c}68 \\
72,5 \\
72\end{array}$ & $\begin{array}{l}49-87 \\
59-84 \\
57-80\end{array}$ \\
\hline A2 & $\begin{array}{l}\text { E. localizada } \\
\text { E. L. avanzada } \\
\text { E. metastásica }\end{array}$ & $\begin{array}{l}49 \\
28 \\
11\end{array}$ & $\begin{array}{l}69 \\
69 \\
69\end{array}$ & $\begin{array}{l}6,3 \\
6,7 \\
4,8\end{array}$ & $\begin{array}{c}70 \\
69,5 \\
69\end{array}$ & $\begin{array}{l}52-80 \\
57-81 \\
60-77\end{array}$ \\
\hline A3 & $\begin{array}{l}\text { E. localizada } \\
\text { E. L. avanzada } \\
\text { E. metastásica }\end{array}$ & $\begin{array}{c}61 \\
6 \\
10\end{array}$ & $\begin{array}{l}68 \\
75 \\
70\end{array}$ & $\begin{array}{c}9,1 \\
5,4 \\
10,4\end{array}$ & $\begin{array}{c}66 \\
75,5 \\
71\end{array}$ & $\begin{array}{l}49-87 \\
68-82 \\
51-83\end{array}$ \\
\hline A4 & $\begin{array}{l}\text { E. localizada } \\
\text { E. L. avanzada } \\
\text { E. metastásica }\end{array}$ & $\begin{array}{c}206 \\
6 \\
15 \\
\end{array}$ & $\begin{array}{l}69 \\
73 \\
79\end{array}$ & $\begin{array}{c}8,1 \\
9 \\
7,1\end{array}$ & $\begin{array}{c}69 \\
71,5 \\
80\end{array}$ & $\begin{array}{l}40-89 \\
59-86 \\
68-91\end{array}$ \\
\hline A6 & $\begin{array}{l}\text { E. localizada } \\
\text { E. L. avanzada } \\
\text { E. metastásica }\end{array}$ & $\begin{array}{c}67 \\
3 \\
14\end{array}$ & $\begin{array}{c}69 \\
74 \\
69,5\end{array}$ & $\begin{array}{c}8,7 \\
9,1 \\
9\end{array}$ & $\begin{array}{l}69 \\
73 \\
70\end{array}$ & $\begin{array}{l}50-88 \\
66-84 \\
52-81\end{array}$ \\
\hline A7 & $\begin{array}{l}\text { E. localizada } \\
\text { E. L. avanzada } \\
\text { E. metastásica }\end{array}$ & $\begin{array}{l}90 \\
24 \\
17\end{array}$ & $\begin{array}{l}72 \\
71 \\
71\end{array}$ & $\begin{array}{c}7,6 \\
6,4 \\
7\end{array}$ & $\begin{array}{l}71 \\
71 \\
71\end{array}$ & $\begin{array}{l}49-89 \\
53-81 \\
56-82 \\
\end{array}$ \\
\hline A8 & $\begin{array}{l}\text { E. localizada } \\
\text { E. L. avanzada } \\
\text { E. metastásica }\end{array}$ & $\begin{array}{l}80 \\
36 \\
21\end{array}$ & $\begin{array}{c}69 \\
69,5 \\
72\end{array}$ & $\begin{array}{c}7 \\
9,1 \\
6,6\end{array}$ & $\begin{array}{l}68 \\
69 \\
72\end{array}$ & $\begin{array}{l}53-84 \\
53-84 \\
56-85\end{array}$ \\
\hline A9 & $\begin{array}{l}\text { E. localizada } \\
\text { E. L. avanzada } \\
\text { E. metastásica }\end{array}$ & $\begin{array}{c}53 \\
7 \\
13 \\
\end{array}$ & $\begin{array}{l}65 \\
71 \\
66 \\
\end{array}$ & $\begin{array}{c}7,2 \\
9,3 \\
9 \\
\end{array}$ & $\begin{array}{l}65 \\
69 \\
64 \\
\end{array}$ & $\begin{array}{l}47-84 \\
60-85 \\
49-82 \\
\end{array}$ \\
\hline A12 & $\begin{array}{l}\text { E. localizada } \\
\text { E. L. avanzada } \\
\text { E. metastásica }\end{array}$ & $\begin{array}{c}55 \\
6 \\
4\end{array}$ & $\begin{array}{c}69,5 \\
70 \\
70\end{array}$ & $\begin{array}{l}5,4 \\
6,1 \\
2,9\end{array}$ & $\begin{array}{c}70 \\
67,5 \\
70,5\end{array}$ & $\begin{array}{l}57-82 \\
63-79 \\
66-73\end{array}$ \\
\hline H.M. & $\begin{array}{l}\text { E. localizada } \\
\text { E. L. avanzada } \\
\text { E. metastásica }\end{array}$ & $\begin{array}{c}27 \\
4 \\
7\end{array}$ & $\begin{array}{l}70 \\
66 \\
73\end{array}$ & $\begin{array}{l}8,9 \\
6,7 \\
8,6\end{array}$ & $\begin{array}{l}72 \\
67 \\
76\end{array}$ & $\begin{array}{l}51-94 \\
57-73 \\
60-81\end{array}$ \\
\hline
\end{tabular}

PE: pacientes evaluables. DS: desviación estándar. Min-Máx.: edad mínima y máxima. H.M.: hospitales militares.

capsular). En nuestro estudio casi la mitad de los pacientes $(47,6 \%)$ presentaron un tacto rectal no sospechoso de tumor y el $84 \%$ fueron clasificados como enfermedad localizada con esta exploración. Al comparar las distintas áreas sanitarias observamos que existen diferencias, algunas importantes, al clasificar los pacientes como tumor no palpable o tumor palpable localizado en la glándula, pero cuando los agrupamos como tumor localizado o localmente avanzado estas diferencias se reducen considerablemente. En la serie de Arrizabalaga y cols. ${ }^{5}$ el $21,1 \%$ se clasificaron como T1, el 45,6\% como T2 y el 33,3\% como T3-T4. Si lo comparamos con los datos del mismo Área
Sanitaria de nuestro estudio observamos que han disminuido al $19 \%$ y al 30\% los pacientes etiquetados como T3-T4 y T2, aumentando al 50\% los casos T1. En la serie de Herranz y cols. ${ }^{6}$ de 613 pacientes con CP diagnosticados mediante biopsia ecodirigida en el periodo 1994-1998 en el H. Gregorio Marañón (Área Sanitaria 1): el 26\% no tenian sospecha al tacto rectal, el $49 \%$ tenían un nódulo localizado a la glándula y el $25 \%$ se clasificaron como localmente avanzados al tacto rectal. Si lo comparamos con los datos del Área 1 de nuestro estudio observamos que los pacientes con tacto rectal normal se elevó al $42 \%$, disminuyendo los casos con nódulo localizado al $44 \%$ y sobre 
todo los pacientes con enfermedad localmente avanzada al 14\%. Basándonos en estas dos comparaciones históricas observamos que estamos diagnosticando más precozmente a los pacientes.

La mitad de los pacientes tenían en el momento del diagnóstico $11 \mathrm{ng} / \mathrm{ml}$ o menos de PSA. Aunque el PSA aislado no es un buen predictor de la extensión de la enfermedad, se considera que la mitad de los pacientes con un PSA entre 4 y $10 \mathrm{ng} / \mathrm{ml}$ tienen enfermedad extracapsular, los pacientes con más de $10 \mathrm{ng} / \mathrm{ml}$ tienen un riesgo aumentado de presentar enfermedad extraprostática y cuando el PSA es superior a $20 \mathrm{ng} / \mathrm{ml} \mathrm{el}$ riesgo supera el $80 \%^{7}$. Al comparar el PSA al diagnóstico en las distintas áreas sanitarias observamos que no existen grandes diferencias entre ellas. Las áreas que presentan una mediana de PSA menor o igual a $10 \mathrm{ng} / \mathrm{ml}$ son las áreas 4, 5, 8, 9 y 12. En el Registro de Tumores de Rotterdam en el año 1995 la mediana de PSA fue de $15,1 \mathrm{ng} / \mathrm{ml}^{8}$.

La ecografía transrectal con la posibilidad de biopsia ecodirigida es la técnica más eficaz para confirmar la sospecha de CP establecida por elevación de PSA o por tacto rectal sospechoso. Esta técnica ha propiciado un llamativo incremento de los diagnósticos de $\mathrm{CP}^{9}$. En el estudio el 93\% de los pacientes fue diagnosticado mediante biopsia transrectal ecodirigida, el 3\% mediante biopsia "ciega" o "digito-dirigida" y el $4 \%$ se diagnosticó de forma incidental por cirugía prostática. Al comparar el método de diagnóstico entre las distintas áreas, todas excepto las áreas 3, 6 y los hospitales militares tienen un porcentaje de biopsia ecodirigida superior al 90\%. En cambio, las áreas 3 y 6 diagnosticaron a un 21\% y un 35\% respectivamente mediante biopsia "ciega" y los Hospitales militares a un $12 \%$ mediante cirugía prostática. Aunque la biopsia transrectal ecodirigida se considera la técnica estándar para el diagnóstico del $\mathrm{CP}$, se admite la biopsia "ciega" cuando los pacientes presentan un tacto rectal sospechoso ${ }^{10}$, sobre todo cuando debido a su expectativa de vida no serían candidatos a cirugía radical.

Debido a la gran heterogeneidad del CP la clasificación histológica que se utiliza actualmente es la de Gleason ${ }^{11}$, basada en la arquitectura tumoral a bajo aumento. Gleason describió 5 patrones tumorales (1-5, de menor a mayor diferenciación). El grado tumoral final es el resultado de la suma (score) de los dos patrones predominantes. Los scores se agrupan en: bien diferenciados (scores 24), moderadamente diferenciados (scores 5-7) y pobremente diferenciados (scores 8-10). En este estudio los más frecuentes fueron el score 6 $(31,7 \%)$ y el score $7(28,9 \%)$ y lógicamente el grado de diferenciación más frecuente fue el moderado (71\%) seguido del pobremente diferenciado (20\%). Al comparar el score de Gleason entre las distintas áreas sanitarias se observa una gran diferencia en el porcentaje de los scores 6 (13\% y 47,9\%) y 7 (6,5\% y $51,6 \%)$, también se observa cuando se agrupan por grados de diferenciación. Esto puede ser debido a varias razones dependientes de: el tumor (heterogeneidad y multicentricidad), la técnica de biopsia (número de muestras, tamaño $\mathrm{y}$ localización de las mismas) y de la interpretación del patólogo (confusión al asignar el grado tumoral, elegir uno u otro grado cuando ninguno de los dos es incorrecto o "bordeline error" y a la variabilidad interobservador que existe al interpretar la biopsia). Si comparamos los datos de nuestro estudio del Área 8 con el control histórico ${ }^{5}$, observamos que han disminuido los tumores bien $\mathrm{y}$ pobremente diferenciados aumentando los moderadamente diferenciados desde un $44,6 \%$ al $59 \%$. Si hacemos lo mismo con los datos históricos del Área $1^{6}$, existe también una disminución de los tumores bien y pobremente diferenciados y un aumento desde un $38 \%$ al $67 \%$ de los tumores moderadamente diferenciados. Esta migración de grado de diferenciación puede deberse a varias razones:

1. Aumento de la experiencia y cambios de criterios en los Servicios de Anatomía Patológica.

2. En el estudio histórico del Área 8 el porcentaje de casos de CP incidentales fue del 36,9\%, mientras que en nuestro estudio disminuyó al 5\%. En el H. Gregorio Marañón (Área 1) en el periodo $1994-97$ el $11,2 \%$ de los casos de CP se diagnosticaron de forma incidental (datos no publicados), mientras que en el estudio sólo supusieron el 4\%. Esto puede justificar la disminución de los tumores bien diferenciados.

3. El aumento de diagnósticos de $\mathrm{CP}$ con tacto rectal no sospechoso puede influir en la disminución de los tumores pobremente diferenciados. 
En el trabajo de Spapen sobre el programa de screening de Rotterdam ${ }^{8}$ los cambios en el tiempo (1989-1995) del grado de diferenciación indican que aumentan los bien diferenciados hasta el $33,6 \%$, se mantienen los moderadamente diferenciados $(35,9 \%)$ y disminuyen los pobremente diferenciados hasta el $23,7 \%$.

En función de los datos aportados por los Investigadores, el 75\% de los pacientes fueron clasificados como enfermedad localizada (T1-2, NO/Nx, $\mathrm{MO} / \mathrm{Mx}$ ), el $12,5 \%$ como enfermedad localmente avanzada (T3-4, NO/Nx, MO/Mx) y el 12,5\% como enfermedad metastásica (T1-4, $\mathrm{N}+\mathrm{o} \mathrm{M}+$ ). Existe una relación entre la edad y el estadio tumoral, los pacientes con enfermedad localizada son un poco más jóvenes que los otros. Nuestros datos son muy similares a los publicados para USA en $1995^{12}$, $77 \%, 13 \%$ y $10 \%$ respectivamente. En la serie de Murphy y cols. ${ }^{13}$ el $21 \%$ de los pacientes con CP en el periodo de tiempo entre 1981 y 1990 presentaban metástasis a distancia. Los programas de screening están produciendo una migración del estadio, disminuyendo el porcentaje de pacientes con metástasis en el momento del diagnóstico. En el European Randomized Study of Screening for Prostate Cancer (ERSSPC) de Rotterdam el $77 \%$ se clasificó como enfermedad localizada, el $21 \%$ como localmente avanzada y sólo el $2 \%$ como metastásica ${ }^{14}$. En este estudio se enfatiza la gran diferencia, estadísticamente significativa, que existe al comparar sus datos con los del registro de tumores de Amsterdam ( $24 \%$ de enfermedad metastásica) que no tiene un programa de screening. Si comparamos los datos históricos ${ }^{5}$ del Área 8 con los de nuestro estudio, el porcentaje de enfermedad metastásica ha disminuido del 32,8\% al 15,3\%. En el Área 1 al comparar con datos históricos ${ }^{15}$ la enfermedad metastásica ha disminuido del $21 \%$ al $13,4 \%$.

En conclusión, la mayoría de los pacientes diagnosticados de CP consultaron por síndrome miccional. La biopsia transrectal ecodirigida se confirma como la técnica de elección para el diagnóstico del CP. Al comparar los datos de nuestro estudio con series históricas de dos áreas sanitarias de la $\mathrm{CM}$, observamos una anticipación diagnóstica ya que los pacientes se diagnostican con menor volumen tumoral al tacto rectal, disminución de los tumores pobremente diferenciados y reducción de los pacientes con enfermedad localmente avanzada o metastásica.

\section{REFERENCIAS}

1. MAGANTO PAVÓN E.: Cáncer de próstata. EMISA. Madrid, 1986.

2. CATALONA WJ, SCOTT WW.: Carcinoma de próstata. En: Walsh, Gittes, Perlmutter, Stamey: Campbell Urología. 5 ed. Ed. Médica Panamericana. Buenos Aires, 1988.

3. HERMANEK P, HUTTER RVP, SOBIN LH, WAGNER G, WITTEKIND CH (eds.).: Atlas TNM. Guía ilustrada de la clasificación TNM/pTNM de los tumores malignos. Tumores urológicos. Springer 1998.

4. GOODMAN CM, CHISHOLM GD.: Presentación y supervivencia en el adenocarcinoma de próstata. Análisis de 438 casos consecutivos estudiados a lo largo de 10 años. Arch Esp Urol 1989; 42 (supl II):117-123.

5. ARRIZABALAGA MORENO M, GARCÍA GONZÁLEZ JI, DÍEZ RODRÍGUEZ JM y cols.: Indicadores epidemiológicos del adenocarcinoma de próstata. Resultados sobre 436 pacientes. Actas Urol Esp 1997; 21: 852-861.

6. HERRANZ AMO F, VERDÚ TARTAJO F, DÍEZ CORDERO JM y cols.: Características clínicas y ecográficas del cáncer de próstata diagnosticado mediante biopsia transrectal. Arch Esp Urol 1999; 52: 1067-1072.

7. CARROL P, COLEY C, MCLEOD D et al.: Prostate-specific antigen best practice policy - Part I: early detection and diagnosis of prostate cancer. Urology 2001; 57: 217-224.

8. SPAPEN SJJC, DAMHUIS RAM, KIRKELS WJ.: Trends in the curative treatment of localized prostate cancer after the introduction of prostate-specific antigen: data from the Rotterdam cancer registry. BJU International 2000; 85: 474-480.

9. HERRANZ AMO F.: Estado actual de la biopsia transrectal ecodirigida de la glándula prostática. Actas Urol Esp 1998; 22: 461-471.

10. RICHIE JP, MURPHY GP, WALTHER P.: Prostate cancer surgical practice guidelines. Oncology 1997; 11: 907-912.

11. GLEASON DF, MELLINGER GT.: The Veterans Administration Cooperative Urological Research Group: prediction of prognosis for prostatic adenocarcinoma by combined histological grading and clinical staging. $J$ Urol 1973; 111: 58-64.

12. METTLIN CJ, MURPHY GP, ROSENTHAL DS, MENCK HR.: The national cancer database report on prostate carcinoma after the peak in incidence rates in the U.S. Cancer 1998; 83: $1679-1684$.

13. MURPHY M, JOHNSTON C, WHELAN P, RIDER L, LLOYD $\mathrm{SN}$.: Changing trends in prostatic cancer. BJU International 1999; 83: 786-791.

14. RIETBERGEN JBW, HOEDEMAEKER RF, KRUGER AEB, KIRKELS WJ, SCHRÖDER FH.: The changing pattern of prostate cancer at the time of diagnosis: characteristics of screen detected prostate cancer in a population based screening study. J Urol 1999; 161: 1192-1198.

15. HERRANZ AMO F, DÍEZ CORDERO JM, VERDÚ TARTAJO F y cols.: Correlación entre el antígeno específico prostático, el grado tumoral y el estadio local con la gammagrafía ósea en el estadiaje de los pacientes con cáncer de próstata. Arch Esp Urol 1997; 50: 253-258.

Dr. F. Herranz Amo

C/ Doctor Esquerdo, 155 $-7^{\circ} 3$

28007 Madrid

(Trabajo recibido el 19 noviembre de 2002) 